\title{
Nuclear DNA content of isolated crypts of background colonic mucosa from patients with familial adenomatous polyposis and sporadic colorectal cancer
}

\author{
S Nakamura, I Kino, S Baba
}

Department of

Pathology, Hamamatsu

University Hospital

S Nakamura

First Department of Pathology

I Kino

and Second Department of Surgery, Hamamatsu

University School of

Medicine, 3600 Handa-

Japan

S Baba

Correspondence to:

Professor S Nakamura,

Department of Pathology,

Department of Pathology,

Hospital, 19-1 Uchimaru,

Mospital, 19-1 Uchinar 020 Japan.

Accepted for publication

15 December 1992 cho, Hamamatsu 431-31,

\begin{abstract}
The DNA content of the upper one third of the crypt epithelium was compared with that of the lower two thirds in the background colorectal mucosa of eight cases of familial adenomatous polyposis (FAP) and eight control cases of sporadic colorectal cancer (SCRC). Intact crypts were isolated by incubating fresh lesion free colorectal mucosa in calcium and magnesium free Hanks' balanced salt solution (CMFH) containing $30 \mathrm{mM}$ EDTA for 30 minutes at $37^{\circ} \mathrm{C}$ and then agitating in CMFH. The crypts were then separated from the lamina propria, fixed in $70 \%$ ethanol and under a dissecting microscope divided manually into upper and lower portions. Each portion was digested with pepsin to obtain a suspension of single nuclei, and smears of the nuclei were stained with 4',6,-diamidino-2-phenylindole dihydrochloride (DAPI). Nuclear DNA was determined using a cytophotometric microscope. Results showed that the DNA content of the epithelium of the upper one third of crypts was diploid in both FAP and SCRC cases, and that proliferative fractions with diploid peaks were present in the lower two thirds of the crypts in both groups. These results support our previous finding that the proliferative compartment of background crypts is confined to the lower two thirds and does not extend to the upper parts of the crypts.
\end{abstract}

(Gut 1993; 34: 1240-1244)

TABLE I Clinicopathological data on eight patients with familial adenomatous polyposis

\begin{tabular}{llll}
\hline No & $\begin{array}{l}\text { Agel } \\
\text { sex }\end{array}$ & $\begin{array}{l}\text { Surgical } \\
\text { procedure }\end{array}$ & Pathological finding \\
\hline 1 & $45 / \mathrm{F}$ & Total colectomy & 1 cancer and 707 adenomas \\
2 & $54 / \mathrm{F}$ & Total colectomy & 1000 adenomas \\
3 & $29 / \mathrm{M}$ & Total colectomy & 4 cancers and 3243 adenomas \\
4 & $29 / \mathrm{F}$ & Total colectomy & 2 cancers and 5094 adenomas \\
5 & $56 / \mathrm{M}$ & Total colectomy & 697 adenomas \\
6 & $22 / \mathrm{F}$ & Total colectomy & 425 adenomas \\
7 & $40 / \mathrm{M}$ & Total colectomy & 121 adenomas \\
8 & $49 / \mathrm{M}$ & Total colectomy & 158 adenomas \\
\hline
\end{tabular}

TABLE II Clinicopathological data on eight patients with sporadic colorectal cancer

\begin{tabular}{llll}
\hline No & Age/sex & Surgical procedure & Pathological findings \\
\hline 1 & $50 / \mathrm{M}$ & Partial colectomy & Advanced cancer of the descending colon \\
2 & $70 / \mathrm{M}$ & Caecectomy & Advanced cancer of the caecum \\
3 & $67 / \mathrm{M}$ & Subtotal colectomy & 17 tubular adenomas and a dysplasia \\
4 & $65 / \mathrm{F}$ & Sigmoidectomy & Advanced cancer of the sigmoid colon \\
5 & $59 / \mathrm{F}$ & Partial colectomy & Early cancer of the transverse colon \\
6 & $64 / \mathrm{M}$ & Right hemicolectomy & Advanced cancer of the ascending colon \\
7 & $62 / \mathrm{F}$ & Right hemicolectomy & Advanced cancer of the ascending colon \\
8 & $60 / \mathrm{M}$ & Sigmoidectomy & Advanced cancer of the sigmoid colon \\
\hline
\end{tabular}

Lipkin proposed that upward expansion of the proliferative compartment of the crypts of the large intestine occurs before adenoma development. ${ }^{1}$ In both familial adenomatous polyposis (FAP) cases and sporadic colorectal cancer (SCRC) cases, morphologically normal epithelial cells with abnormal DNA synthesis are reported to be present in upper part of crypts. ${ }^{2-6}$

In previous studies on the morphogenesis of colonic adenomas in FAP cases using a complete serial sectioning technique, we found that adenomas begin to develop in the proliferative compartment of the lower crypts in the form of atypical epithelial clusters, 'buds of adenoma'. We did not, however, detect any upward expansion of the proliferative compartment in the background crypts. We also examined whether upward expansion actually occurred in the background solonic mucosa by ex vivo autoradiography. ${ }^{8}$ Our results confirmed that the distribution of labelled cells in FAP cases was exactly the same as that in SCRC cases: the proliferative compartment was located in the lower two thirds of the crypts and no abnormality of cell kinetics was seen in either FAP or SCRC cases. ${ }^{9}$

There are many reports of studies on the phase of DNA synthesis in normal colonic crypts, ${ }^{10-12}$ but none on the DNA distribution in epithelial cells in colonic crypts. In this study we examined the distribution of nuclear DNA in crypts of background mucosa from FAP and SCRC cases by cytophotometry.

\section{Methods}

CASES STUDIED

Studies were made on eight cases of FAP and eight control cases, seven with SCRC and one with multiple colonic adenomas. Tables I and II show the clinicopathological findings in these eight FAP cases and eight SCRC cases, respectively.

\section{CRYPT ISOLATION}

The method of Arai and Kino was used for crypt isolation. ${ }^{13}$ Specimens of the colorectal mucosa were obtained just after their surgical excision. Normal mucosa at least $5 \mathrm{~cm}$ from carcinomas in SCRC cases and apparently normal mucosa between multiple adenomas in FAP cases were separated from the submucosa with scissors. The mucosa was cut into $2 \mathrm{~mm}$ squares with a razor incubated at $37^{\circ} \mathrm{C}$ for 30 minutes in calcium and 


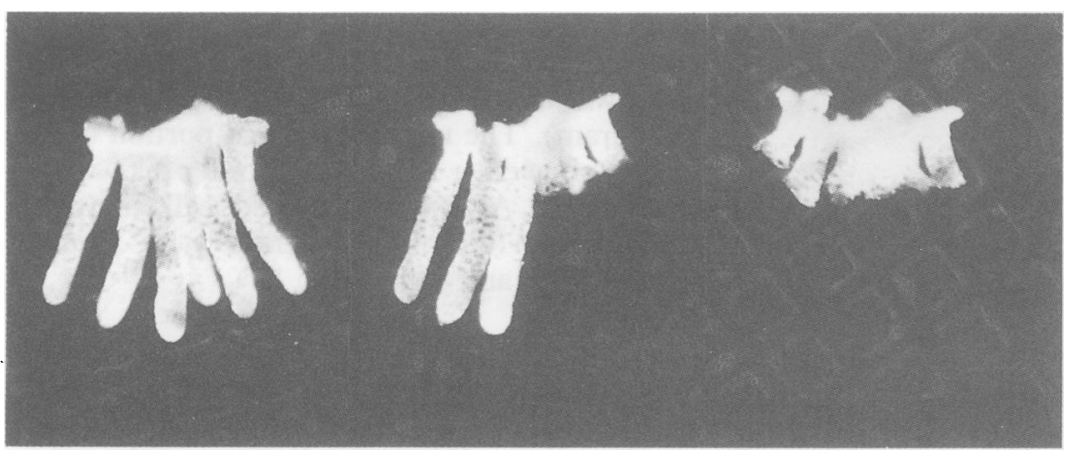

Figure 1: Procedure for crypt division. Intact and straight crypts were placed on cover glasses under which copper grids for electron microscopy were mounted on microscope slides. Left; six intact crypts connected with surface epithelium. Centre; three crypts divided into upper one third and lower two thirds portions. Right; three other crypts divided similarly. The copper grid was used as a scale in division of crypts.

magnesium free Hanks' balanced salt solution (CMFH) containing $30 \mathrm{mM}$ EDTA and then stirred in CMFH. The crypts separated from the lamina propria mucosa within about 30 minutes and were promptly fixed in $70 \%$ ethanol.

\section{DIVISION OF CRYPTS}

Straight, intact crypts were divided at the junction of the upper one third and lower two thirds under a dissecting microscope, as follows: isolated crypts were put on cover glasses and these were placed on microscopic slides under which copper grids (as used for electron microscopy) had been mounted. The grids were used for assessing the position most appropriate for bisection of the crypts (Fig 1). Fine needles used by ophthalmologists for surgical suture (Davis and Geck Ins, USA) with wooden stems were used to manipulate and divide the isolated crypts under a dissecting microscope. Fifty to 60 crypts from each case were divided into upper and lower portions.

\section{PROCESSING FOR CYTOPHOTOMETRY}

Each upper one third and lower two third portion was incubated with $0.05 \%$ pepsin

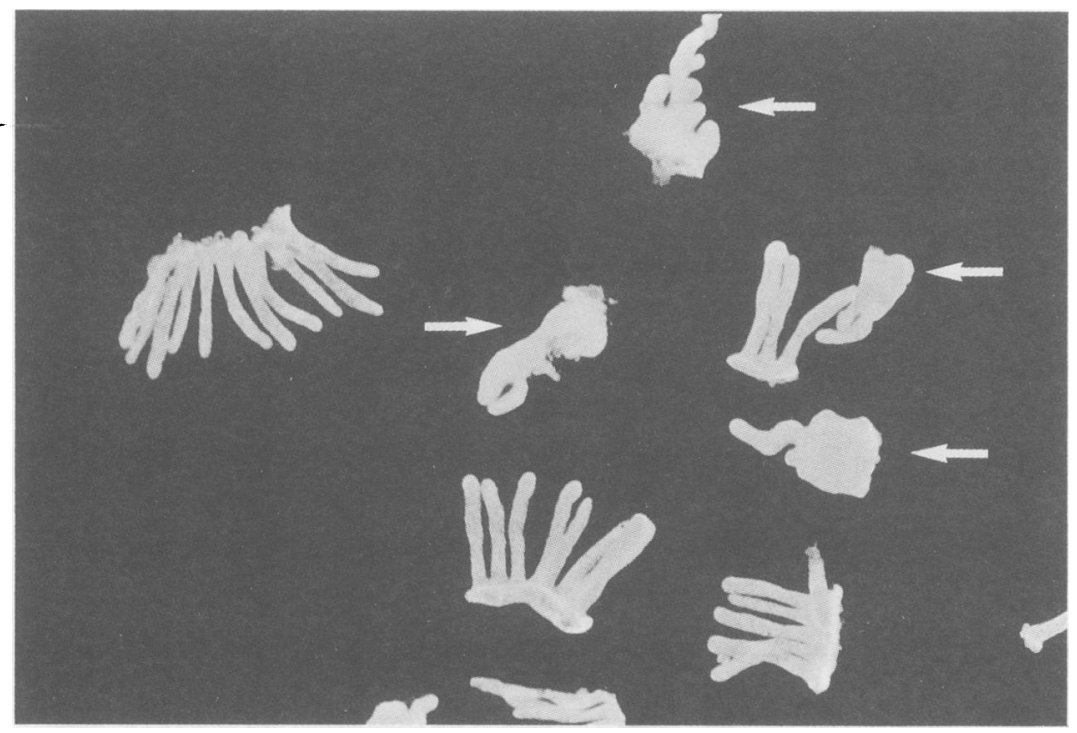

Figure 2: Normal crypts are finger shaped and are connected by surface epithelium. Irregular shaped larger crypts with branching are adenomas (arrows).

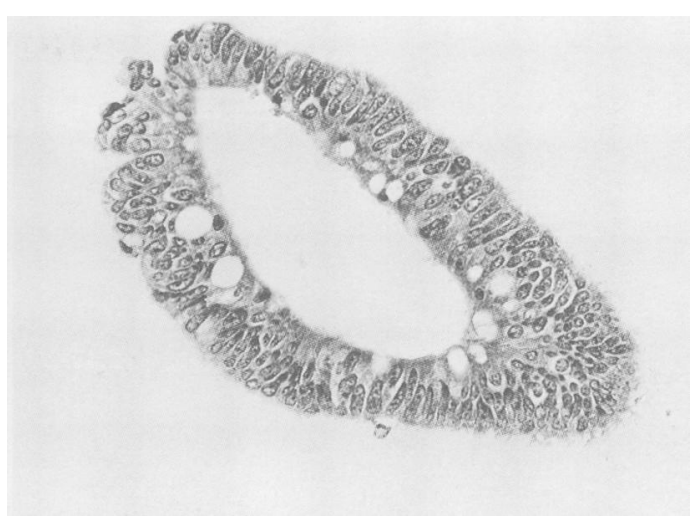

Figure 3: Histological section of an adenomatous crypt identified by dissecting microscopy. Note complete absence of lamina propria and basement membrane. (Haematoxylin and eosin stain original magnification $\times 100$.)

(Sigma, USA) adjusted to $\mathrm{pH} 2 \cdot 0$, for five minutes at $37^{\circ} \mathrm{C}$, washed twice with $0.2 \mathrm{M}$ TRIS chloride buffered saline, and aspirated and ejected several times through a disposable syringe ( $1 \mathrm{ml}$, Nipro, Tokyo) with a 27 gauge needle. The single nuclei separated in this way were filtered through $37 \mu \mathrm{m}$ nylon mesh (Tokyo Screen, Tokyo) and smeared on microscope slides using a Cytospin 3 (Shandon Inc, England). The nuclei on the slides were stained with $1 \mu \mathrm{g} / \mathrm{ml}$ of $4^{\prime}, 6$-diamidino-2-phenylindole dihydrochloride (DAPI) (Sigma, USA) in $0.2 \mathrm{M}$ TRIS chloride buffered saline for one hour at room temperature in the dark. Stained nuclei were examined in a fluorescence cytophotometric microscope (OSP-1, Olympus, Tokyo). For each of the 16 pairs of histograms shown, 200-212 nuclei were examined.

\section{Results}

Colonic crypts were easily separated from lamina propria mucosa by agitating fragments of mucosa obtained by incubation with EDTA. In the FAP cases, minute adenomas and single gland adenomas, ${ }^{7}$ which could not be detected by naked eye, were easily recognised under the dissecting microscope (Fig 2). We confirmed that these crypts were adenomatous in paraffin sections stained with haematoxylin and eosin (Fig 3). Even in FAP cases normal crypts could be selected for study under a dissecting microscope.

The DAPI stained nuclei on smear slides were round or oval with a uniform size and shape. Nuclei that overlapped each other were not used for DNA measurements (Fig 4).

Figure 5 shows eight pairs of DNA histograms of crypt epithelia of FAP cases. The numbers of the histograms correspond with those of the cases shown in Table I. Each top half of a pair shows the DNA distribution in the upper one third, and the bottom half shows that in the lower two thirds. The abscissa represents the amount of DNA and the ordinate shows the number of nuclei. In all eight cases, the lower two third portion shows a distinct diploid peak, which corresponds to the $G_{0}$ or $G_{1}$ phase in the 


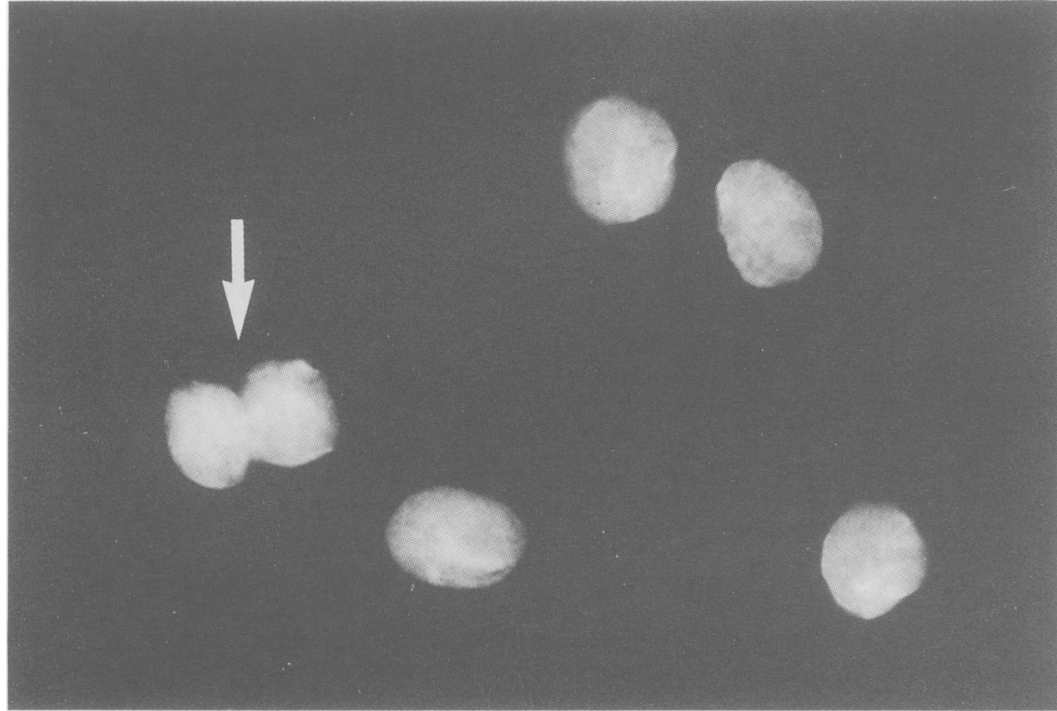

Figure 4: Isolated nuclei stained with DAPI seen by fluorescent microscopy. Nuclei that overlapped each other (arrow) were eliminated from DNA measurements. (Original magnification $\times 400$.) in the eight SCRC cases. As seen in the FAP cases, a proliferating compartment was seen in the lower two third portion of the crypts (lower histogram). The upper one third portion of the crypts showed a distinct diploid peak (upper histogram). A few non-diploid cells between diploid and tetraploid fractions were seen in cases $3,4,5$, and 8 .

\section{Discussion}

According to Lipkin, during the development of colonic adenomas, several stages of abnormalities in cell kinetics are seen between normal and adenomatous crypts while maintaining a normal cellular morphology. In the first stage, crypt cells that can synthesise DNA spread beyond the normal proliferative compartment into the upper part of the gland. Next, these abnormally proliferating cells accumulate in the surface epithelium of the mucosa, and finally adenomas appear in the upper part of the mucosa. ' Studies by in vitro autoradiography showed that in both FAP cases and SCRC cases, cells with abnormal DNA synthesis actually spread in the upper part and surface of the crypts of the background mucosa. ${ }^{1-6}$

We have studied the morphogenesis of minute adenomas in FAP cases by complete serial sectioning of apparently normal colonic mucosa and have found that adenomas developed in the middle part of normal crypts, sprouting into lamina propria mucosa as 'buds of adenomas'.? We propose that a transformed cell develops in the proliferative compartment of the lower part of the crypt, divides several times, and moves
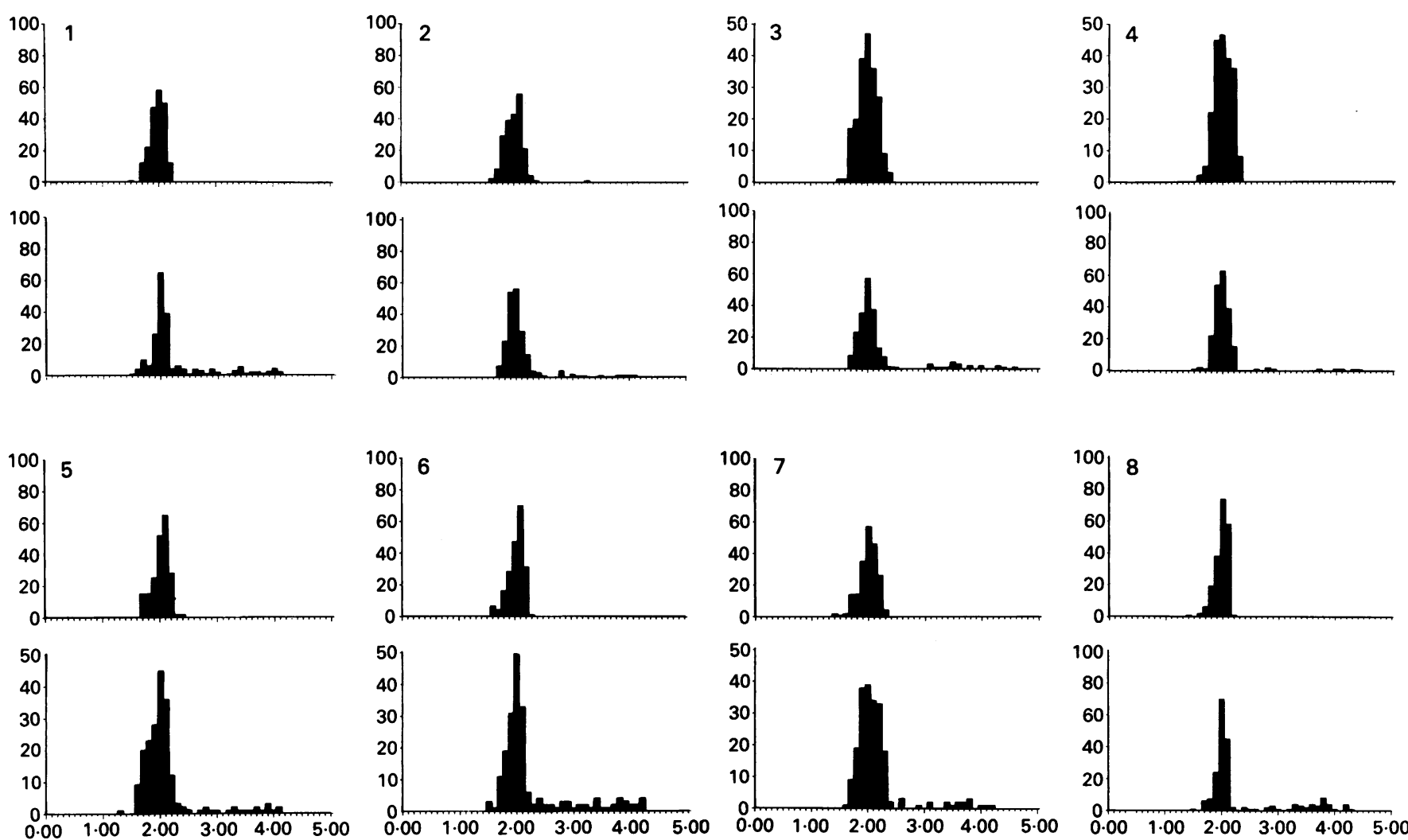

Figure 5: Pairs of DNA histograms of crypt epithelia in eight FAP cases. The numbers of pairs correspond with those of the cases shown in Table I. The upper histogram of each pair shows the DNA distribution in the upper one third of the crypts, and the lower histogram shows that of the lower two third portion. The abscissa represents the amount of DNA and the ordinate indicates the number of nuclei. 

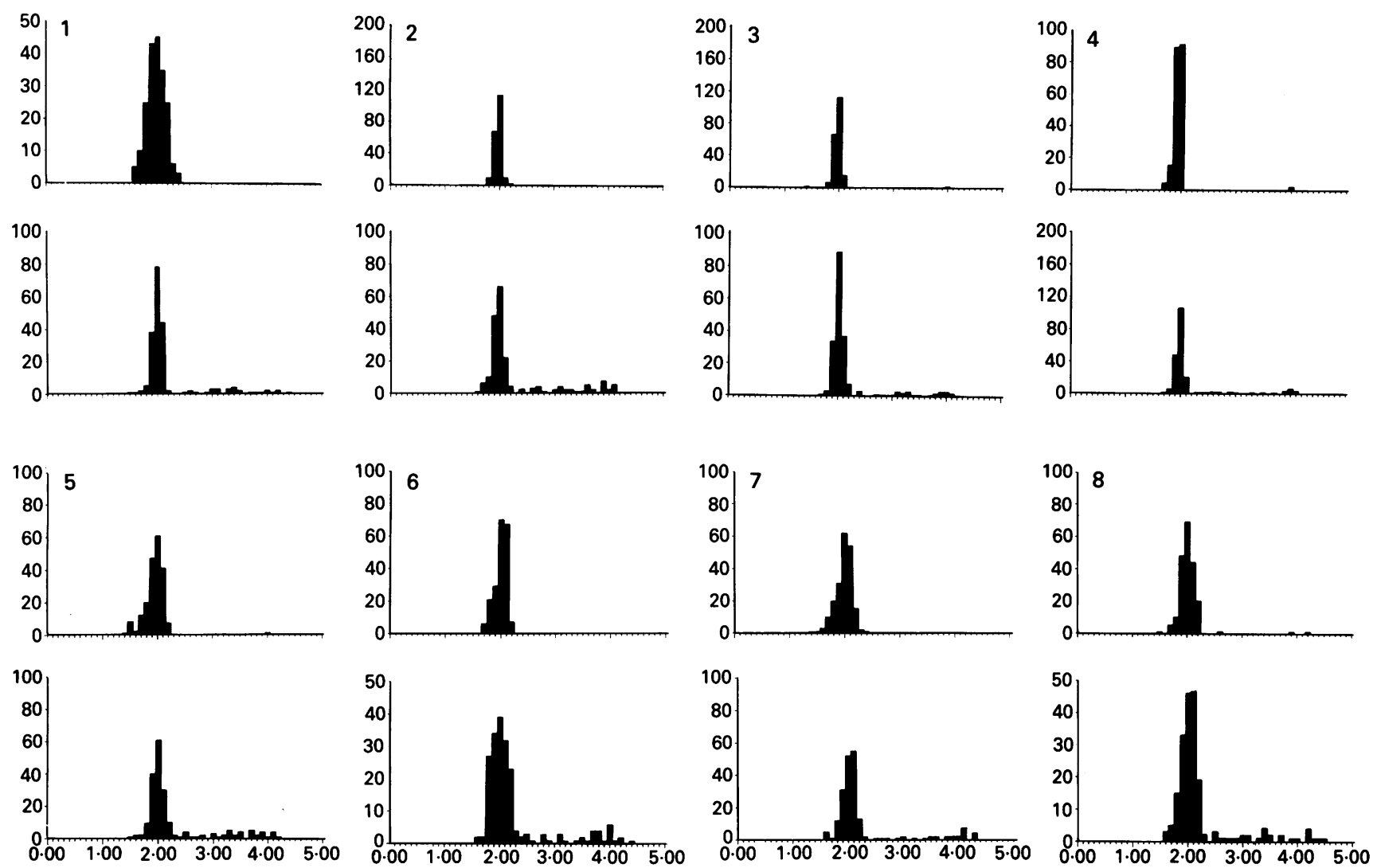

Figure 6: DNA histograms of crypt epithelia in the eight SCRC cases. The abscissa and the ordinate are the same as for Fig 5.

upwards with migration of the normal crypt epithelium. In the middle part of the crypt, a cluster of transformed cells breaks through the crypt basement membrane and is identified as an adenoma bud. The upwards shift of the proliferating compartment is not necessary for the development of adenomas buds, even in FAP cases. To discover if an upward shift of the proliferative compartment actually takes place, we examined the distribution of labelled cells and the labelling index of the background colonic mucosa in FAP and SCRC cases by ex vivo autoradiography. Results showed that the distributions of labelled cells and the labelling indices were exactly the same in FAP and SCRC cases. $^{9}$

Well oriented crypts cut longitudinally along their entire length in histological sections are necessary to evaluate the position of the labelled cells accurately by autoradiography. Specimens obtained by endoscopy and treated with in vitro autoradiography must be less than $1 \mathrm{~mm}$ thick and $5 \mathrm{~mm}$ wide to obtain a reliable labelling index,${ }^{14}$ and are usually composed of obliquely cut crypts in histological sections. To avoid these limitations of size and orientation, we used ex vivo autoradiography, which allowed us to select well oriented crypts in the large histological sections $(20 \times 40 \mathrm{~mm}){ }^{8}$ We found that differences in the methods used for analyses of cell kinetics are major causes of differences in results on whether the proliferative compartment is normal.

By isolating the crypts from lamina propria, exact measurements of the DNA content of the crypts was possible. ${ }^{13}$ is 16 Intact and straight crypts fixed in $70 \%$ ethanol were easily selected under a dissecting microscope. It is possible that a few cells or nuclei were damaged at the site of crypt bisection and damaged nuclei would not have been measured. As there would have been equal numbers of damaged nuclei, however, in both groups (upper one third and lower two thirds) of crypt fractions we feel that this is not likely to be a significant distorting factor in the analysis.

Aberrant crypts that were abnormally large or showed branching, which are considered to be minute adenomas or hyperplastic crypts, ${ }^{17}$ could be omitted in this experiment.

No cells synthesising DNA are present in the upper one third and surface epithelium of normal colonic crypts. ${ }^{811-13}$ There is no other report, however, of comparisons of the DNA contents of the upper one third and lower two thirds of crypt epithelium. This study showed that all nuclei in the upper one third of crypts have a diploid DNA content in both FAP cases and SCRC cases. This study thus confirmed our theory that the proliferating compartment of the background colonic crypts is confined to the normal position, even when the crypts affect the initial stage of development of adenomas.

This work was supported by a Grant in Aid for Cancer Research from the Ministry of Education, Science and Culture. The authors thank Professor Tetuichiro Muto and Dr Yoshiro Kubota, Department of Surgery, Faculty of Medicine University of Tokyo, for permission to examine case 1 of FAP and Tsuneji Noguchi for for permission to examine case 1 of FAP and Tsuneji Noguchi for photography. We are also grateful to Dr Jonathan Sheffield and Dr their instructive suggestions.

1 Lipkin M. Phase 1 and phase 2 proliferative lesions of colonic epithelial cells in diseases leading to colonic cancer. Cancer 1974; 34: 878-88.

2 Deschner EE, Lipkin M. Proliferative patterns in colonic mucosa in familial polyposis. Cancer 1975; 35: 413-8.

3 Iwama T, Utsunomiya J, Sasaki J. Epithelial cell kinetics in Iwama T, Utsunomiya J, Sasaki J. Epithelial cell kinetics in the crypts
$7: 230-4$. 
4 Lipkin M, Blattner WA, Gardner EJ, Burt RW, Lynch H Deschner E, et al. Classification and risk assessment of individuals with familial polyposis, Gardner's syndrome, and familial non-polyposis colon cancer from $\left[{ }^{3} \mathrm{H}\right]$ Thymidine labeling patterns in colonic epithelial cells. Cancer Res 1984; 44: 4201-7.

5 Risio M, Lipkin M, Candelaresi GL, Bertone A, Coverlizza S, Rossini FP. Correlations between rectal mucosa cell proRossini FP. Correlations between rectal mucosa cell prononfamilial neoplasia of the large intestine. Cancer Res 1991 , 51: 1917-21.

6 Paganelli GM, Biasco G, Santucci R, Brandi G, Lalli AA Miglioli $M$, et al. Rectal cell proliferation and colorecta cancer risk level in patients with nonfamilial adenomatou polyps of the large bowel. Cancer 1991; 68: 2451-4.

7 Nakamura S, Kino I. Morphogenesis of minute adenomas in familial polyposis coli. $\mathcal{F}$ Natl Cancer Inst 1984; 73: 41-9.

8 Nakamura S, Kino I, Baba S. Ex vivo autoradiography of the human gastrointestinal tract: A new approach to cell kinetic studies of surgically removed tumour-bearing organs. $\mathcal{f} p n \mathcal{F}$ Cancer Research (Gann) 1983; 74: 116-21.

9 Nakamura S, Kino I, Baba S. Cell kinetics analysis of background colonic mucosa of patients with intestinal neoplasms by ex vivo autoradiography. Gut 1988; 29: 997-1002.

10 Shorter RG, Spencer RJ, Hallenbeck GA. Kinetic studies of the epithelial cells of the rectal mucosa in normal subjects and patients with ulcerative colitis. Gut 1966; 7: 593-6.

11 Bleiberg $\mathrm{H}$, Mainguet $\mathrm{P}$, Galand $\mathrm{P}$. Cell renewal in familia polyposis: comparison between polyps and adjacent healthy mucosa. Gastroenterology 1972; 63: 240-5.

12 Bleiberg $H$, Galand $P$. In vitro autoradiographic determination of cell kinetic parameters in adenocarcinomas and adjacent healthy mucosa of the human colon and rectum. Cancer Res 1976; 36: 325-8.

13 Arai T, Kino I. Morphometric and cell kinetic studies of normal human colorectal mucosa. Comparison between the proximal and the distal large intestine. Acta Pathol fpn 1989, proximal and $725-30$.

14 Steel GG, Bensted JPM. In vitro studies of cell proliferation in tumours - I. Critical appraisal of methods and theoretica considerations. Eur $\mathcal{F}$ Cancer 1965; 1: 275-9.

15 Bjerknes $M$, Cheng $H$. Methods for the isolation of intac epithelium from the mouse intestine. Anat Rec 1981; 199: 565-74.

16 Cheng H, Bjerknes M, Amar J. Methods for the determination of epithelial cell kinetic parameters of human colonic epithelium isolated from surgical and biopsy specimens. Gastroenterology 1984; 86: 78-85.

17 Roncucci L, Medline A, Bruce WR. Classification of aberran crypt foci and microadenomas in human colon. Cancer Epidemiology Biomarkers and Prevention 1991; 1: 57-60 